\title{
Câncer de pulmão: atualização da terapêutica cirúrgica
}

\author{
Eduardo H. Saito,,$^{*}$ Rogério Rufino, ${ }^{2}$ Cláudia Henrique da Costa, ${ }^{2}$ Nelson B. Pimentel, ${ }^{3}$ Luiz Carlos Aguiar, ${ }^{4}$ \\ Domenico Capone ${ }^{2}$
}

\begin{abstract}
Resumo
O câncer de pulmão é uma doença agressiva e seu diagnóstico precoce direciona o tratamento para uma maior chance de cura. Dados gerais da literatura apontam que uma minoria de pacientes (cerca de 30\%), portadores de carcinoma não pequenas células, limitadas ao pulmão, beneficiam-se do tratamento cirúrgico curativo. Desta forma, todo esforço para um diagnóstico precoce deve ser realizado. O objetivo deste artigo de atualização é abordar o tema proposto de uma forma geral, ressaltando as informações mais relevantes para profissionais da área de saúde. Exames cada vez mais sofisticados podem ser utilizados na prática médica, assim como técnicas cirúrgicas mais evoluídas e inovações de quimioterapia/radioterapia complementam o avanço para uma maior sobrevida.
\end{abstract}

Descritores: Neoplasias pulmonares; Oncologia; Diagnóstico; Estadiamento de neoplasias; Terapia combinada; Cirurgia torácica videoassistida.

\section{Abstract \\ Lung cancer: update surgical therapy}

The lung cancer is an aggressive disease and early diagnosis guides its treatment to a greater chance of cure. Literature data indicates that a minority of patients, about 30\%, with non-small cell lung cancer, benefits from curative surgical treatment, so every effort for early diagnosis should be made. As discussed here, sophisticated tests may be used for earlier detection in medical practice, and more advanced surgical techniques and innovations in chemotherapy/radiation therapy may allow for an extended survival.

Keywords: Lung neoplasms; Medical oncology; Diagnosis; Neoplasm staging; Combined modality therapy; Thoracic surgery, video-assisted.
1. Disciplina de Cirurgia Torácica. Faculdade de Ciências Médicas. Universidade do Estado do Rio de Janeiro. Rio de Janeiro, RJ, Brasil.

2. Disciplina de Pneumologia. Faculdade de Ciências Médicas. Universidade do Estado do Rio de Janeiro. Rio de Janeiro, RJ, Brasil.

3. Serviço de Pneumologia. Hospital Universitário Pedro Ernesto. Universidade do Estado do Rio de Janeiro. Rio de Janeiro, RJ, Brasil.

4. Disciplina de Anatomia Patológica. Faculdade de Ciências Médicas. Universidade do Estado do Rio de Janeiro. Rio de Janeiro, RJ, Brasil.

*Endereço para correspondência:

Secretaria de Pneumologia, HUPE, UERJ

Boulevard 28 de Setembro, 77, 20 andar

Rio de Janeiro, RJ, Brasil. CEP: 22551-030

E-mail: pneumo.uerj@gmail.com

Revista HUPE, Rio de Janeiro, 2015;14(Supl. 1):87-96

doi: 10.12957/rhupe.2015.17933

Recebido em 03/06/2015. Aprovado em 15/07/2015.

\section{Resumen}

\section{Cáncer de pulmón: actualización terapia} quirúrgica

El cáncer de pulmón es una enfermedad agresiva y el diagnóstico precoz dirige su tratamiento hacia una mayor posibilidad de curación. Datos generales de la literatura indican que una minoría de pacientes, aproximadamente el 30\% de los pacientes con carcinoma de células no pequeñas, limitadas al pulmón, se benefician del tratamiento quirúrgico curativo, por lo que se debe realizar todos los esfuerzos posibles para el diagnóstico precoz. El objetivo de este artículo de actualización es abordar el tema propuesto de una forma general, resaltando las informaciones más relevantes para profesionales del área de salud. Exámenes cada vez más sofisticados pueden ser utilizados en la práctica médica, así como técnicas quirúrgicas más desarrolladas e innovaciones en quimioterapia/radioterapia complementan el avance hacia una mayor prolongación de la vida.

Palabras clave: Neoplasias pulmonares; Oncología médica; Diagnóstico; Estadificación de neoplasias; Terapia combinada; Cirugía torácica asistida por video. 


\section{Artigo de revisão}

\section{Introdução}

O câncer de pulmão passou de doença pouco frequente no início do século XX à oitava causa de morte por neoplasia. No Brasil, é o segundo câncer mais comum no sexo masculino (superado apenas pelo de próstata) e o quarto mais frequente no sexo feminino. Segundo dados levantados pelo Instituto Nacional do Câncer (INCA), em 2011, o risco estimado para câncer de pulmão era de 18 para cada 100 mil pessoas do sexo masculino e de dez para 100 mil no sexo feminino, sendo que há variações de prevalência entre as regiões brasileiras, com as maiores taxas nas regiões Sul e Sudeste. ${ }^{1}$

O fator de risco mais associado ao câncer de pulmão é o tabagismo, o que permite planejar e sonhar com o controle (ou redução) desta neoplasia com medidas de prevenção ao hábito. ${ }^{1}$

Outros fatores de risco estão associados ao câncer de pulmão, como a radiação ionizante e a inalação das substâncias inorgânicas sílica, asbesto e produtos químicos como cromo, níquel e arsênico. ${ }^{2}$

No Brasil, embora não haja uma estatística precisa, acredita-se que os números de nódulos pulmonares sejam proporcionalmente maiores, devido à elevada incidência de tuberculose. Neste caso particular, o estado do Rio de Janeiro apresenta especial relevância, uma vez que o coeficiente de incidência de casos novos de tuberculose, segundo estatística de 1998 do Ministério da Saúde(MS), era de 83,7 por 100 mil habitantes; quase o dobro da média nacional, de 44 por 100 mil habitantes. ${ }^{1}$

\section{Manifestações clínicas}

Aproximadamente 5\% dos cânceres pulmonares primários são assintomáticas quando se faz o diagnóstico. ${ }^{1,2}$

Os sintomas broncopulmonares são causados pelo tumor devido à irritação, à ulceração ou à obstrução, podendo haver combinação dessas manifestações em um brônquio. Podem haver também complicações sépticas (infecciosas) do parênquima distal ao tumor, sendo fundamental lembrar que toda pneumonia classificada como comunitária pode ser secundária a um tumor obstruindo o brônquio, e por isso tem de ser acompanhada até a resolução radiológica. Caso a melhora não ocorra, deve-se proceder à broncoscopia de inspeção da árvore brônquica. O sintoma mais comumente associadoé a tosse, mas também pode ocorrer hemoptise, dor torácica, dispneia e febre. ${ }^{2}$

Os sintomas extrapulmonares e intratorácicos são causados pela extensão direta ou metástases locorre- gional e linfonodal, envolvendo pleura parietal, parede torácica e estruturas mediastinais ou nervos contíguos. Eles ocorrem em até $15 \%$ dos pacientes. ${ }^{2}$

A paralisia do nervo laríngeo recorrente é mais comum à esquerda do que à direita, e a síndrome de obstrução da veia cava superior pode acontecer em até $5 \%$, especialmente no oatcell (pequenas células). A rouquidão associada à neoplasia pulmonar é sinal de mau prognóstico. A paralisia do nervo frênico é rara e o envolvimento do plexo braquial e de parede torácica acontece em menor frequência. ${ }^{2}$

A síndrome de Claude-Bernard-Horner ocorre mais tardiamente e é secundária à destruição (ou estimulação) do plexo simpático por um tumor de lobo superior. ${ }^{2}$

O derrame pleural sem desvio do mediastino pode sugerir que esse derrame seja maligno, pelo comprometimento linfonodal que mantém o mediastino fixo. ${ }^{2}$

Os sintomas extratorácicos não metastáticos ocorrem em $2 \%$ dos casos e são citados como síndromes paraneoplásicas (Tabela 1). As neuromiopatias são as mais frequentes manifestações não metastáticas extratorácicas. Se pesquisadas, podem ser encontradas em até 15\% dos pacientes com neoplasia de pulmão. Outras alterações podem ocorrer, como: miopatia carcinomatosa (síndrome miastênica de Eaton Lambert) e neuropatia periférica. ${ }^{2}$

A manifestação musculoesquelética mais comum é o baqueteamento digital, que pode estar associado à osteoartropatia hipertrófica. ${ }^{2}$

A acantose nigricanse o sinal de Leser-Trélat (aparecimento abrupto, com rápido aumento em número e tamanho, de múltiplas ceratoses seborreicas associado à presença de uma neoplasia) podem acontecer como manifestações extratorácicas dos cânceres de pulmão. ${ }^{2}$

A síndrome de Trousseau é uma desordem da coagulação sanguínea adquirida, que resulta em trom-

\section{Tabela 1. Relação tumor e manifestações metabólicas.}

\begin{tabular}{|l|c|}
\hline \multicolumn{1}{c|}{ Manifestações } & $\begin{array}{c}\text { Classificação histológica tumoral } \\
\text { (mais frequente) }\end{array}$ \\
\hline $\begin{array}{l}\text { Síndrome de Cushing } \\
\text { Secreção inapropri- } \\
\text { ada do hormônio } \\
\text { antidiurérico (SIADH) }\end{array}$ & $\begin{array}{c}\text { Tumor de pequenas células } \\
\text { Hipercalcemia }\end{array}$ \\
\hline $\begin{array}{l}\text { Ginecomastia } \\
\text { Hipocalcemia }\end{array}$ & Tumor escamoso pequenas células \\
\hline
\end{tabular}


boflebite migratória. $^{2}$

As síndromes hematológicas incluem alterações nas linhas celulares da hematopoiese e nos fatores da coagulação. A anemia crônica, a trombocitose e a trombocitopenia podem ser observadas. ${ }^{2}$

As lesões glomerulares paraneoplásicas se traduzem, na maior parte dos casos, por proteinúria maciça e síndrome nefrótica. ${ }^{2}$

Os sintomas metastáticos extratorácicos resultantes das metástases extratorácicas correspondem a 3\% a 6\% das manifestações apresentadas pelos pacientes com câncer de pulmão. Os sintomas neurológicos mais comuns são hemiplegia, a mudança de personalidade, a confusão mental e as alterações visuais. Lesões metastáticas para ossos, com apresentações de fraturas espontâneas patológicas e aspectos líticos (rarefação óssea) provocam dor intensa. As metástases para fígado causam alterações, aumento do volume hepático, dor e icterícia colestática. ${ }^{2}$

Apresentam características radiológicas conclusivas de benignidade a calcificação central, em pipoca, lamelar ou a calcificação total. A tomografia computadorizada de tórax de alta resolução (TCAR) com cortes de 1,0 $\mathrm{mm}$ a 1,5 $\mathrm{mm}$ auxiliam no diagnóstico de nódulos de até $2 \mathrm{~cm}$ de diâmetro com maior precisão e auxiliam na identificação de calcificação e do aspecto da calcificação no nódulo. ${ }^{3}$

\section{Imagens no câncer de pulmão}

Se não ocorrer crescimento tumoral em três anos ou mais, a lesão é provavelmente benigna, embora o adenocarcinoma (bronquíolo-alveolar) possa ser uma exceção a esta regra. Outro aspecto a ser considerado é o tempo de dobra (aumento do volume). Aumento de $25 \%$ no diâmetro representa a duplicação de volume (ex.: tumores de 1,0 cm + 25\% ficam com diâmetro de $1,3 \mathrm{~cm}$, tendo dobrado de volume; tumores de $2,0 \mathrm{~cm}+$ $25 \%$ ficam com $2,5 \mathrm{~cm}$ e o dobro do volume). O tempo de dobra para as lesões malignas é muito variável, de 37 a 465 dias. ${ }^{2.3}$

Os nódulos são considerados suspeitos de malignidade devido à sua morfologia (espiculado, lobulado, atenuação não homogênea, extensão pleural) ou quando apresentam aumento maior que $20 \mathrm{UH}$ na TCAR após a injeção do contraste (para nódulos maiores que $1 \mathrm{~cm}$ ), em associação a outros dados clínicos (Tabela 2).,4

As imagens obtidas pela radiografia de tórax podem ser resultantes da expansão tumoral ou devido às obstruções brônquicas, que levam à atelectasia e/ ou causam infecção. Também é possível observar o envolvimento da pleura (derrame pleural) ou dos linfonodos (linfadenopatias), invasão de arcos costais, vértebras, pericárdio (derrame pericárdio) ou paralisia diafragmática. ${ }^{2}$

Em geral, quando o tumor é visualizado na radiografia de tórax, já se passaram até $3 / 4$ da história natural da evolução do tumor. ${ }^{2}$

Com a introdução da tomografia computadorizada multislice (várias hélices), o radiologista passou a ter de lidar com um número muito maior de imagens, o que pode levar à identificação de mais nódulos do que antigamente. A identificação de nódulos com auxílio de programas de computação do tipo computer-aided detection $(\mathrm{CAD})$ comprovadamente aumenta o número de nódulos detectados. Esta técnica está disponível em poucos serviços e é dispendiosa. Apresenta também resultados falso-positivos. Outra forma de aumentar a detecção de nódulos pulmonares é com a utilização da técnica de projeção de intensidade máxima (MIP), com cortes espessos. ${ }^{3,5}$

O carcinoma escamoso apresenta lesões centrais em $65 \%$ dos casos, podendo obstruir o brônquio e evoluir com atelectasia, consolidação (pneumonia obstrutiva), cavitação (10 a 20\%) e abscessos., ${ }^{2,6}$

No adenocarcinoma encontra-se frequentemente (60\% a 70\% das vezes) na forma de massa periférica. A cavitação é rara. Quando a imagem é nodular, a borda costuma ser mal definida.,

O carcinoma indiferenciado de grandes células apresenta-se como lesões periféricas em $60 \%$ dos casos e costuma ser maior do que $4 \mathrm{~cm}$. A cavitação é rara.,

$\mathrm{O}$ indiferenciado de pequenas células-tumores frequentemente apresenta metástases para linfonodos hilares e mediastinais ( $78 \%$ dos casos). Assim, é comum observar alteração na região hilar. Em poucos casos (13\%), o tumor é descoberto com o alargamento mediastinal sem visualização da lesão tumoral.2,6

Tabela 2. Relação idade e nódulo solitário pulmonar.

\begin{tabular}{|c|c|}
\hline Faixa etária do paciente & $\begin{array}{c}\text { Probabilidade de malignidade } \\
\text { (história pregressa de tabagismo) }\end{array}$ \\
\hline $35-39$ anos & $3 \%$ \\
\hline $40-49$ anos & $15 \%$ \\
\hline $50-59$ anos & $43 \%$ \\
\hline 60 anos & $50 \%$ \\
\hline
\end{tabular}

Fonte: American Journal of Roentgenology. ${ }^{3}$ 


\section{Artigo de revisão}

\section{Métodos diagnósticos}

O exame de escarro é extremamente útil, podendo identificar células tumorais em muitos pacientes. Dependendo da experiência do patologista, a positividade fica entre $45 \%$ a $90 \%$ dos casos. O número de amostras de escarro também se correlaciona com a positividade do exame: três amostras aumentam a positividade para $69 \%$ dos casos. ${ }^{7}$

O antígeno carcinoembrionário (CEA), quando elevado, tende a demonstrar neoplasia avançada e pode estar associado também com o aumento do tumor oatcell. Os marcadores tumorais que podem ser solicitados incluem os hormônios like - big ACTH, B2 microglobulina e várias poliaminas., ${ }^{2,7}$

A tomografia computadorizada (TC) de tórax deve ser solicitada para todos os pacientes em investigação de neoplasia pulmonar. Para facilitar a investigação, pode-se solicitar que os cortes sejam feitos até o andar superior do abdômen, para pesquisa de metástases no fígado e suprarrenais. $\mathrm{O}$ uso de contraste facilita o estudo do nódulo e ajuda na avaliação de invasão de estruturas vitais. A TC é útil na demonstração de linfonodo calcificado e de cavitação da lesão. É um bom exame para avaliar invasão de corpo vertebral.,7

Através da TC, pode-se avaliar linfonodos com alargamento do mediastino superior. Os linfonodos mediastinais são considerados normais se apresentarem diâmetro menor ou igual a $1 \mathrm{~cm}$, exceto os linfonodos subcarinais, que são inferidos como anormais apenas quando crescem mais do que $1,5 \mathrm{~cm} .{ }^{2,3,7}$

A TC de tórax consegue avaliar bem os derrames pleurais, mas não é um exame de eleição para o estudo de invasão para a parede.?

A ressonância magnética é uma ferramenta boa para o estudo da gordura mediastinal, do fluxo sanguíneo nos vasos mediastinais e da ocorrência de invasão da parede. Ela geralmente é mais solicitada para avaliação de pacientes com tumor de Pancoast (tumor do sulco superior). No entanto, também pode ser usada naqueles pacientes com alergia a contraste. As desvantagens deste exame são a pobre resolução espacial, a dificuldade de interpretação de imagens na presença de artefatos e a difícil visualização das linfadenomegalias. $2,7,8$

A ultrassonografia (US) de abdômen possui sensibilidade semelhante à TC na avaliação do envolvimento hepático. Atualmente, a US de abdômen pode ser complementada com a US transesofágica (EUS) - que avalia os linfonodos mediastinais, para-aórticos, subcarinais, paraesofagianos e do ligamento pulmonar -, e com a US endobrônquica (EBUS), que consegue visualizar bem os linfonodos do mediastino pré e paratraqueais. Na presença de aumento destes linfonodos se realiza a punção aspirativa para confirmação do diagnóstico. ${ }^{9}$

As anormalidades genéticas no câncer de pulmão têm sido cada vez mais estudadas. As famílias RAS e ERB-B estão presentes com maior frequência nas neoplasias de não pequenas células e a família MYC é mais encontrada em pacientes com neoplasia de pequenas células. $\mathrm{O}$ tratamento dos pacientes com câncer tem sido modulado de acordo com a presença ou ausência destas alterações. ${ }^{10}$

A broncoscopia é realizada de rotina, exceto nas lesões pequenas periféricas, que não apresentam adenomegalia hilar ou mediastinal.Em até $25-30 \%$ é possível a visualização do tumor e a realização de biópsia. A maior sensibilidade ocorre nos carcinomas de não pequenas células. Alguns broncoscopistas preferem realizar o procedimento no momento da anestesia, quando o paciente é intubado (se estes forem candidatos à cirurgia), pois pode haver presença de lesão endobrônquica sincrônica em torno de $1 \%$ dos casos. A ultrassonografia endobrônquica com punção aspirativa transbrônquica (EBUS-TBNA) é eficaz para avaliação das adenomegalias paratraqueais à esquerda e à direita, subcarinal, hilar e intrapulmonar. ${ }^{9}$

A aspiração transtorácica percutânea está indicada para os casos de tumor clinicamente não ressecável, de alto risco clínico ou quando o paciente recusa a ressecção. A taxa de resultado falso-negativo é de 15 a $25 \%$ dos casos. Além disso, as amostras podem ser inadequadas para citologia em cerca de $27 \%$ dos casos. ${ }^{2,9}$

Os derrames pleurais não diagnosticados pela citologia ou através da biópsia pleural utilizando a agulha de Cope representam as maiores indicações do procedimento de toracoscopia, pois permite visualizar a pleura e identificar implantes pleurais. Este exame também é útil na avaliação dos linfonodos mediastinais, principalmente os da estação inferior, vistos no EUS, no estudo dos linfonodos da janela aortopulmonar e na verificação da extensão tumoral para a pleura visceral, além de permitir a biópsia direta da massa, quando a toracotomia estiver contraindicada., ${ }^{2,9}$

A mediastinoscopia é o exame escolhido para abordagem de linfonodos mediastinais, que podem ser: padrão de Carlens (1959); estendida cervical de Ginsberg (1989); anterior de Chamberlain (1966) ou uma combinação dessas abordagens para linfonodos vistos na TC. A realização de videomediastinoscopia com abertura 
progressiva para esvaziamento linfonodal mediastinal via cervical (VAMLA) também é uma abordagem possível. É sempre bom lembrar que a TC e o PET-CT só complementam a indicação para mediastinoscopia e não são procedimentos de substituição.9,11

O EUS-FNA avalia a presença de adenomegalias das cadeias subcarinal, paraesofagiana, do ligamento pulmonar, da janela aortopulmonar e para-aórtica. A sensibilidade, o valor preditivo negativo e a acurácia deste método para doença N2/N3 foram de 74\%, 73\% e $85 \%$, respectivamente. ${ }^{9}$

\section{Investigação para metástases distantes}

A TC de tórax deve ser sempre associada à tomografia de andar superior do abdômen. Metástases para suprarrenal (SR) são frequentes, mas se o aumento da glândula não for significativo, não ultrapassando $2 \mathrm{~cm}$, pode sugerir hiperplasia ou adenoma. Apenas quando verificamos tumor acima de $3 \mathrm{~cm}$ é que o diagnóstico de um tumor metastático se torna mais provável. De qualquer maneira, na prática, em ambos os casos, devese esclarecer o diagnóstico etiológico através de biópsia por agulha. ${ }^{12}$

A TC de crânio de rotina é apenas útil em uma pequena parcela de pacientes assintomáticos (0,8\%). Os protocolos atuais orientam a TC de crânio nos adenocarcinomas e em qualquer tumor não adenocarcinoma em fase avançada (estágio III), N2 conhecido ou nos tumores com extensão além da pleura visceral, se a ressecção for considerada., ${ }^{2,12}$

Sempre que houver elevação de fosfatase alcalina e da LDH, ou quando o paciente se queixar de dor óssea, a cintilografia deverá ser realizada para o estadiamento.,2,12

A tomografia por emissão de pósitron associada à tomografia computadorizada (PET-CT) pode ser realizada para verificação de lesões metabólicas que absorvam o radiomarcador 16 desoxifluorglicose (16 FDG). Os valores da captação são medidos e, sendo elevados, caracterizam o estado de hipermetabolismo da lesão, próprios das doenças neoplásicas, bem como de doenças inflamatórias (sarcoidose nodular) e infecciosas (tuberculoma, histoplasmomas). Este exame apresenta sensibilidade e especificidade superior à TC para o estadiamento tumoral, sendo, respectivamente, no PET-CT, $85 \%$ e $90 \%$, enquanto na TC, $61 \%$ e $79 \% .12,13$

\section{Estadiamento do câncer de pulmão}

As propostas para a sétima edição do estadiamento do câncer de pulmão foram apresentadas para o Ameri- can Joint Committee on Cancer (AJCC) e para a Union International of Control of Cancer (UICC), em 2007, e aprovadas por estas instituições, tendo entrado em vigor no mês de janeiro de 2010 (Tabelas 3 e 4). ${ }^{14}$

\section{Tratamento cirúrgico}

A ressecção cirúrgica é o tratamento-padrão para os pacientes com neoplasia de não pequenas células e que estejam nos estágios I e II. Além disso, todo nódulo pulmonar solitário deve ser ressecado, exceto aqueles que possuírem características benignas, ou quando a condição clínica do paciente contraindique o procedimento cirúrgico. ${ }^{15,16}$

A classificação de operabilidade deve incluir o risco clínico da cirurgia (doença cardíaca e pulmonar) e o risco da remoção do parênquima pulmonar. ${ }^{16}$

A doença cardiopulmonar, nos seus vários graus, geralmente por consequência do fumo, representa o fator clínico mais significativo na determinação de operabilidade e é a maior causa de morbidade e mortalidade pós-operatória. Cerca de 90\% dos pacientes com câncer têm doença pulmonar obstrutiva crônica (DPOC) e até 20\% apresentam deficiência grave na função pulmonar. ${ }^{16}$

A avaliação pulmonar pré-operatória deve incluir a espirometria e a estimação da função pulmonar no pós-operatório. A toracotomia é geralmente contraindicada quando o volume expiratório forçado no primeiro segundo $\left(\mathrm{VEF}_{1}\right)$ e a capacidade vital forçada (CVF) são menores do que 30\% dos valores preditivos para o paciente. Quando o $\mathrm{VEF}_{1}$ for igual a $60 \%$ (ou maior que o valor preditivo), o paciente será considerado capaz de tolerar a ressecção. Dados intermediários necessitam de avalição mais detalhada, que podem incluir a medida da difusão de monóxido de carbono e do consumo máximo de oxigênio (Quadro 3). O consumo máximo de oxigênio $\left(\mathrm{VO}_{2}\right.$ máximo é utilizado para determinar a ressecabilidade em paciente com espirometria limítrofe. Considera-se favorável a operabilidade quando a capacidade de difusão ao monóxido de carbono estiver superior a $40 \%$ do previsto e o $\mathrm{VO}_{2}$ máximo maior que $20 \mathrm{ml} / \mathrm{kg} / \mathrm{min} .^{2,16}$

Quando a espirometria é limítrofe para a ressecção pulmonar, deve-se solicitar cintilografia pulmonar perfusional/ventilatória, com quantificação por campos pulmonares e, assim, realizar uma análise, identificando os campos pulmonares e a sua participação na função pulmonar. ${ }^{16}$

A definição final de ressecabilidade é feita durante a 


\section{Artigo de revisão}

Tabela 3. Especificações do estadiamento TNM.

\begin{tabular}{|c|c|}
\hline Tx & $\begin{array}{l}\text { Tumor provado pela presença de células neoplásicas nas secreções broncopulmonares ou em lavados } \\
\text { broncoalveolares, porém não identificado pela radiografia ou broncoscopia, ou qualquer tumor que } \\
\text { não possa ser localizado. }\end{array}$ \\
\hline T0 & Nenhuma evidência de tumor primário. \\
\hline Tis & Carcinoma in situ. \\
\hline $\mathrm{T} 1$ & $\begin{array}{l}\text { Tumor com menos de } 3 \mathrm{~cm} \text { no seu maior diâmetro, circundado por pleura ou tecido pulmonar em } \\
\text { toda a sua extensão, sem evidência de invasão proximal a um brônquio lobar ao exame endoscópico. }\end{array}$ \\
\hline T1a & Tumor $\leq 2 \mathrm{~cm}$. \\
\hline T1b & Tumor $>2 \mathrm{~cm} \mathrm{e} \leq 3 \mathrm{~cm}$. \\
\hline $\mathrm{T} 2$ & $\begin{array}{l}\text { Tumor }>3 \mathrm{~cm} \text { e } \leq 7 \mathrm{~cm} \text {, ou qualquer uma das seguintes características: } \\
\text { - invade a pleura visceral; } \\
\text { - provoca atelectasia ou pneumonite crônica obstrutiva que não envolva todo o pulmão; } \\
\text { - acomete o brônquio principal a mais de } 2 \mathrm{~cm} \text { da carina principal. }\end{array}$ \\
\hline $\mathrm{T} 2 \mathrm{a}$ & Tumor $>3 \mathrm{~cm} \mathrm{e} \leq 5 \mathrm{~cm}$ \\
\hline $\mathrm{T} 2 \mathrm{~b}$ & Tumor $>5 \mathrm{~cm} \mathrm{e} \leq 7 \mathrm{~cm}$. \\
\hline T3 & $\begin{array}{l}\text { Tumor }>7 \mathrm{~cm}(\mathrm{~T} 3>7 \mathrm{~cm}) \text {, com: } \\
\text { - invasão da parede torácica (incluindo tumores do sulco superior), do diafragma, do pericárdio ou } \\
\text { pleura mediastinal, sem invadir estruturas viscerais, grandes vasos ou corpo vertebral }\left(\mathrm{T}_{\text {inv }}\right) \text {; } \\
\text { - tumores localizados no brônquio principal a menos de } 2 \mathrm{~cm} \text { da carina principal }\left(\mathrm{T} 3_{\text {centr }}\right) \text {; } \\
\text { - tumores com atelectasia ou pneumonite obstrutiva de todo o pulmão }\left(\mathrm{T} 3_{\text {centr }}\right) \text {; } \\
\text { - nódulos-satélites no mesmo lobo }\left(T 3_{\text {satél }}\right) \text {. }\end{array}$ \\
\hline T4 & $\begin{array}{l}\text { Tumor de qualquer tamanho invadindo mediastino, comprometendo coração, grandes, vasos, traqueia, } \\
\text { esôfago, corpo vertebral ou carina ( } 4_{\text {inv }} \text { ): } \\
\text { - nódulos em um lobo ipsilateral diferente (T4 }{ }_{\text {nód. ips }} \text { ). }\end{array}$ \\
\hline $\mathrm{Nx}$ & Metástase linfodonais não demonstráveis. \\
\hline NO & Ausência de metástases linfonodais. \\
\hline N1 & $\begin{array}{l}\text { Metástases linfodonais para região peribrônquica, linfonodos hilares ipsilaterais ou ambos, incluindo } \\
\text { extensão direta (cadeias } 10,11 \text { ). }\end{array}$ \\
\hline N2 & Metástase para linfonodos mediastinais ipsilaterais ou subcarinas (cadeia 2, 4, 5, 6, 7, 8, 9 homolaterais). \\
\hline N3 & $\begin{array}{l}\text { Metástase para linfonodos contralaterais, mediastinais ou hilares ou linfonodos cervicais ou pré- } \\
\text { escalênicos, ipsi ou contralaterais. }\end{array}$ \\
\hline $\mathrm{Mx}$ & Metástases não demonstradas. \\
\hline MO & Ausência de metástases. \\
\hline M1a & 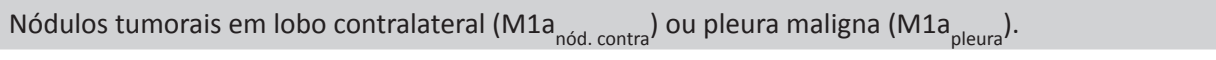 \\
\hline M1b & tástases a distância. \\
\hline
\end{tabular}

Fonte: Journal of Thoracic Oncology. ${ }^{14}$

toracotomia/CTVA. São consideradas contraindicações para ressecção os seguintes achados: metástases pleurais, extenso acometimento de linfonodo mediastinal (N3), extensão direta do tumor para órgãos vitais (T4). ${ }^{15,16}$

O cirurgião deve tentar realizar a ressecção completa do tumor e de todo envolvimento regional brônquico e de nódulos linfáticos mediastinais, incluindo a ressecção de estruturas adjacentes envolvidas por extensão direta do tumor primário (ex.: parede torácica, pericárdio). A ressecção extramúsculo parietal (tipo gaiola de passarinho) de Ribeiro-Netto é uma opção menos invasiva que a ressecção das costelas para aqueles 
Tabela 4. Estadiamento TNM de tumores de pulmão não pequenas células.

\begin{tabular}{|c|c|c|c|c|c|}
\hline$T / M$ & Subgrupos & No & N1 & N2 & N3 \\
\hline $\mathrm{T} 1$ & $\begin{array}{l}\text { T1a } \\
\text { T1b }\end{array}$ & $\begin{array}{l}\text { IA } \\
\text { IA }\end{array}$ & $\begin{array}{l}\text { IIA } \\
\text { IIA }\end{array}$ & $\begin{array}{l}\text { IIIA } \\
\text { IIIA }\end{array}$ & $\begin{array}{l}\text { IIIB } \\
\text { IIIB }\end{array}$ \\
\hline T2 & $\begin{array}{l}\text { T2a } \\
\text { T2b }\end{array}$ & $\begin{array}{l}\text { IB } \\
\text { IIA }\end{array}$ & $\begin{array}{l}\text { IIA } \\
\text { IIB }\end{array}$ & $\begin{array}{l}\text { IIIA } \\
\text { IIIA }\end{array}$ & $\begin{array}{l}\text { IIIB } \\
\text { IIIB }\end{array}$ \\
\hline T3 & $\begin{array}{l}\text { T3>7cm } \\
\text { T3inv } \\
\text { T3satél }\end{array}$ & $\begin{array}{l}\text { IIB } \\
\text { IIB } \\
\text { IIB }\end{array}$ & $\begin{array}{l}\text { IIIA } \\
\text { IIIA } \\
\text { IIIA }\end{array}$ & $\begin{array}{l}\text { IIIA } \\
\text { IIIA } \\
\text { IIIA }\end{array}$ & $\begin{array}{l}\text { IIIB } \\
\text { IIIB } \\
\text { IIIB }\end{array}$ \\
\hline T4 & $\begin{array}{l}\text { T4inv } \\
\text { T4nód.ips }\end{array}$ & $\begin{array}{l}\text { IIIA } \\
\text { IIIA }\end{array}$ & $\begin{array}{l}\text { IIIA } \\
\text { IIIA }\end{array}$ & $\begin{array}{l}\text { IIIB } \\
\text { IIIB }\end{array}$ & $\begin{array}{l}\text { IIIB } \\
\text { IIIB }\end{array}$ \\
\hline M1 & $a / b$ & IV & IV & IV & IV \\
\hline
\end{tabular}

Fonte: Journal of Thoracic Oncology. ${ }^{14}$

tumores que não invadem as costelas (Figura 1). ${ }^{17}$

Em alguns casos, o diagnóstico de tumor somente é realizado durante o procedimento cirúrgico amplo, com a presença de um patologista e realização de biópsia de congelação. Os procedimentos que podem ser realizados são pneumectomia, bilobectomia, lobectomia, segmentectomia e ressecção em "cunha". 15-17

O acesso mais comum é a incisão posterolateral (5o ou $6^{\circ}$ espaço intercostal) com ressecção sistemática dos nódulos linfáticos, com análise histológica por "congelação". 15,16

A ressecção pulmonar mais comum para o câncer de pulmão é a lobectomia. A perda funcional que ocorre imediatamente após a ressecção é geralmente maior do que o previsto, entretanto, após um período de convalescência, a função pulmonar melhora aos níveis previstos pelos testes de função pulmonar. Uma variante da lobectomia convencional é a lobectomia "em manga", retirada do lobo pulmonar com um segmento de brônquio intermediário à direita ou da carina secundária à esquerda e reimplante do brônquio do lobo não comprometido. ${ }^{15,16}$

A pneumectomia é realizada quando a lobectomia não consegue oferecer uma ressecção completa. Para este procedimento, o cirurgião deve estar ciente da reserva cardiopulmonar, para evitar o risco de eventos pulmonares agudos (insuficiência respiratória) e cardíaca (infartos, isquemia e arritmias) no pós-operatório. Três procedimentos distintos de pneumectomia podem ser realizados: pneumectomia clássica modificada (acrescenta a ligadura intrapericárdica dos vasos pulmonares); pneumectomia supra-aórtica à esquerda e pneumectomia em manga traqueal..$^{15,16}$
Figura 1. Ressecção tipo "gaiola de passarinho", de RibeiroNetto.

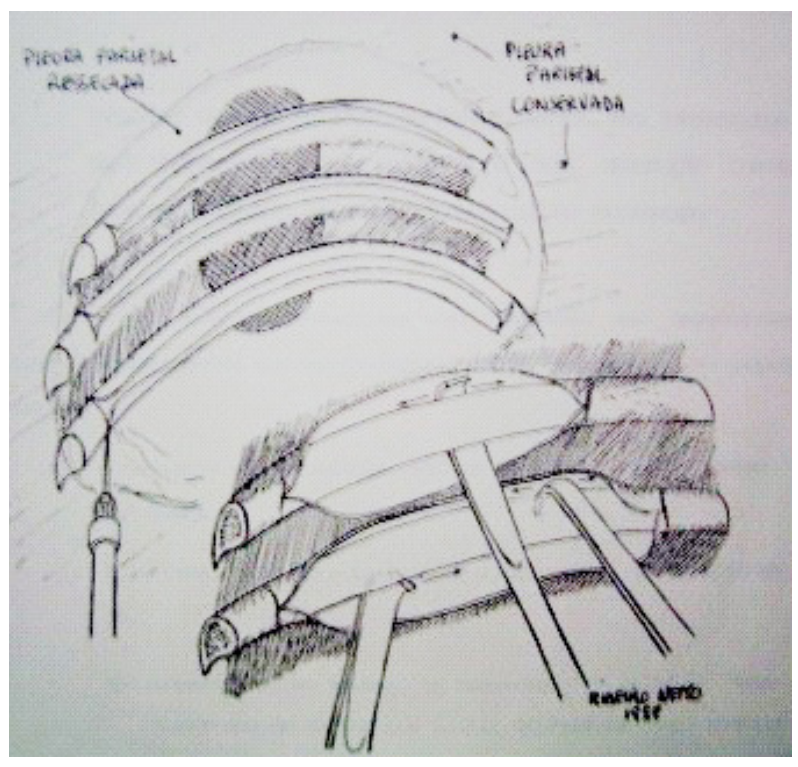

No pós-operatório de uma pneumectomia, o cirurgião pode optar por manter o paciente sem dreno; com dreno fechado e sua abertura a cada $2 / 2$ horas ou uma vez por dia, dependendo da posição do mediastino na radiografia de tórax e com drenagem balanceada. ${ }^{15,16}$

As ressecções "sub-lobares", como segmentectomia anatômica ou não anatômica e a ressecção em cunha, são realizadas em pacientes sem boa reserva pulmonar, apesar de se ter um risco de recorrência local, quando comparadas à lobectomia. Os estudos atuais estão demonstrando que as segmetectomias anatômicas (com seç̧ão em separado dos vasos e brônquio do segmento pulmonar) possuem o risco de recorrência local semelhante às ressecções tipo lobectomia. ${ }^{18}$

A ressecção pulmonar com toracectomia ou com ressecção do diafragma ou do pericárdio podem ser indicadas nos tumores T3.

A cirurgia torácica videoassistida (CTVA) é muito indicada para as nodulectomias de lesões periféricas e, se na análise de congelação da biópsia se confirmar a malignidade, faz-se a lobectomia por CTVA ou pela toracotomia. $^{19-21}$

A CTVA é uma técnica minimamente invasiva que diminui a morbidade cirúrgica, incluindo a dor, e é particularmente vantajosa para os pacientes com comorbidades clínicas significativas. Esta diminuição da morbidade pode reforçar o cumprimento da terapia adjuvante. Estudos com grande séries multiinstitucionais relatam a segurança e a eficácia 
(sobrevida) da lobectomia por CTVA para pacientes no estágio Ia e Ib com tumores não pequenas células, sendo esta realizada em rotina por diversas instituições em todo o mundo. ${ }^{19-24}$

\section{Resultados da ressecção/prognóstico}

O prognóstico se dá de acordo com o estágio da doença e, por sua vez, deve considerar o tratamento mais adequado.

\section{Estágio I}

A sobrevida da lobectomia com dissecção sistemática dos linfonodos e com a análise final (congelação e parafina) dos linfonodos hilar e mediastinal sem acometimento neoplásico em três anos é de $85 \%$ e em cinco anos de $80-85 \%$.

Não é recomendada nenhuma radioterapia ou quimioterapia após completa ressecção do estágio Ia.

No estágio Ib, muitos indicam a quimioterapia adjuvante quando o tumor é maior do que $5 \mathrm{~cm}$ de diâmetro. ${ }^{19-23}$

\section{Estágio II}

A ressecção em bloco dos linfonodos segmentar, lobar, interlobar, hilar e a dissecção sistemática dos linfonodos mediastinais devem ser realizadas para excluir envolvimento neoplásico metastático. A sobrevida em cinco anos é de $40 \%$ a 50\%, com frequência de recorrência de $50 \%$ ou mais. A maioria das recorrências é devido às metástases a distância.

Radioterapia e/ou quimioterapia adjuvante são indicadas e podem reduzir a incidência local e regional de recorrência. ${ }^{19-23}$

\section{Estágio IIIa}

É o carcinoma localmente avançado (N2) e está associado com prognóstico relativamente ruim, mesmo após a cirurgia radical (a ressecção total do tumor, dos tecidos invadidos e dos linfonodos envolvidos). Pacientes com T3N1 (invadindo parede), apesar da ressecção completa, apresentam sobrevida em cinco anos próxima de $40 \%$.

Protocolos multimodais, incluindo quimioterapia e/ou radioterapia neoadjuvante e cirurgia para pacientes N2 estão em avaliação. Há comprovação de aumento de sobrevida quando se encontra resposta patológica completa após a terapia neoadjuvante (ausência de células neoplásicas na peça cirúrgica). ${ }^{19-23}$

Estágio IIIb
Tumores no estágio IIIb (T4 ou N3) podem ser considerados ressecáveis. Nos $\mathrm{T} 4$ achados durante a toracotomia, a ressecção pode ser avaliada quando não há comprometimento metastático dos linfonodos mediastinais. Na pneumectomia com carinectomia em manga a taxa de sobrevida em cinco anos é próxima de $20 \% .^{19-23}$

\section{Estágio IV}

Não são cirúrgicos, exceto os pacientes com metástase única cerebral ressecável. Nestes casos, realiza-se inicialmente a ressecção da metástase cerebral e, em outro tempo, a ressecção pulmonar com linfadenectomia.19-23

\section{Novas técnicas}

Em 2009, cirurgiões da Universidade George Washington (EUA) realizaram 100 lobectomias pulmonares por cirurgia robótica assistida consecutivas e tiveram um tempo médio operatório de 216 +/- 27 minutos. Sem mortalidade operatória. Não tiveram conversão para toracotomia. A mediana da internação foi de quatro dias. As principais complicações foram: fibrilação atrial (13 pacientes), atelectasia (cinco pacientes), fuga de ar prolongada (quatro pacientes), embolia pulmonar (três pacientes), derrame pleural (três pacientes) e pneumonia (um caso). A mortalidade em 30 dias foi de $3 \%$. No seguimento no período de 32 meses um faleceu de recidiva da neoplasia e quatro tiveram metástases a distância. ${ }^{25}$

Radioterapia ablativa estereotáxica (SABR) surgiu como uma das opções de tratamento radical para a fase I do câncer de pulmão não pequenas células (NSCLC), principalmente em pacientes clinicamente inoperáveis. ${ }^{26}$

Em um banco de dados multicêntrico do Japão de mais de 2.000 pacientes tratados com SABR para a fase I do NSCLC, em três anos, a taxa de sobrevida global (SG) e a taxa de sobrevida específica de doença do total de pacientes foi de $72 \%$ e $85 \%$, respectivamente. ${ }^{26}$

\section{Resultados do grupo da UERJ com a técnica CTVA}

Trinta e cinco pacientes com neoplasia pulmonar foram operados por CTVA com registro pleno de dados de maio de 2009 a junho de 2015:

a) sete lobectomias inferiores esquerdas, quatro lobectomias superiores esquerdas, cinco lobectomias superiores direitas, quatro lobectomias médias, quatro lobectomias inferiores direitas, uma seg- 
mentectomia anterior esquerda, uma segmentectomia anterior direita, uma bilobectomia inferior direita e duas pneumectomias direitas (total de 29 pacientes);

b) seis pacientes tiveram a CTVA convertida para toracotomias (uma lesão do brônquio, quatro sangramentos e uma invasão de pleura);

c) o tamanho do tumor $-1,5 \mathrm{~cm}$ a $10,0 \mathrm{~cm}$;

d) o comprimento da incisão - 4,5 cm a 7,5 cm;

e) o tempo de cirurgia - 3,5 a 5 horas;

f) o número de grampeadores cirúrgicos - seis a 13 cargas;

g) tempo de hospitalização - quatro a 16 dias;

h) morbidade - um paciente teve 14 dias de fuga de ar e outro seis dias de fuga de ar; um terceiro paciente apresentou infecção urinária;

i) mortalidade - um paciente faleceu no $12^{\circ}$ dia pós-operatório por obstrução intestinal por brida e embolia pulmonar;

j) diagnóstico histológico - 22 adenocarcinomas, seis carcinomas epidermoides, dois tumores carcinoides típicos, 1 caso de tuberculose (BK), dois carcinomas neuroendócrinos pouco diferenciados, um hamartoma e um nódulo fibroatelectásico.

\section{Conclusão}

Apesar da neoplasia pulmonar ser agressiva e não apresentar um rastreamento precoce daqueles pacientes portadores desta patologia em uma fase inicial (assintomáticos), os avanços no desenvolvimento de análise histológica e molecular, de medicamentos, radioterapia, técnica cirúrgica e cuidados de terapia intensiva estão proporcionando uma terapêutica mais individualizada e com melhores resultados, além de uma maior sobrevida com melhoria na qualidade de vida. A valorização e reconhecimento da medicina paliativa tem sido fundamental na inserção dos pacientes com doença mais avançada.

A pesquisa científica e a conduta terapêutica conduzida com uma equipe multidisciplinar é fundamental para o avanço no diagnóstico, estadiamento e tratamento desses pacientes. A literatura nos mostra quantos trabalhos científicos estão sendo conduzidos em todos esses aspectos. Há esperanças de se ter descobertas interessantes seguindo as pesquisas da neoplasia da mama.

\section{Referências}

1. Instituto Nacional do Câncer/ Ministério da Saúde: Estimativa
2013. Incidência de câncer no Brasil. Rio de Janeiro. INCA. In: 2014 www2.inca.gov.br.wcm/connect/inca/portal/home

2. Blum GM. Clinical presentation of lung câncer. In: Shields TW [et al]. General ThoracicSurgery. Philadelphia: Lippincott William \& Wilkins 2009.p. 1351-1359.

3. Belmandil-Aubry C, Raffy P, Yang W, et al. Aided Detection of Solid Lung Nodules on Follow-Up MDCT Screening: Evaluation of Detection, Tracking, and Reading Time. AJR Am J Roentgenol. 2007;189(4):948-955.

4. World Health Organization Classification of Tumors. Pathology and Genetics of Tumors of the Lung, Pleura, Thymus and Heart. Disponivel em: http://www.iarc.fr/en/publications/pdfs-online/pat-gen/bb10/. Acesso em: 20 July 2015.

5. Remy-Jardin M, Remy J, Giraud F, et al. Pulmonary nodules: detection with thick-section spiral CT versus conventional CT. Radiology. 1993;187(2):513-520.

6. De Wever W, Verschakelen J, Coolen J. Role of imaging in diagnosis, staging and follow-up of lung cancer. Curr Opin Pulm Med. 2014;20(4):385-392.

7. Finigan JH, Kern JA. Lung cancer screening: past, present and future. Clin Chest Med. 2013;34(3):365-371.

8. Wang YX, Lo GG, Yuan J, et al. Magnetic resonance imaging for lung cancer screen. J Thorac Dis. 2014;6(9):1340-1348.

9. Colella S, Vilmann P, Konge L, et al. Endoscopic ultrasound in the diagnosis and staging of lung cancer. Endosc Ultrasound. 2014;3(4):205-212.

10. Popper HH, Ryska A, Tímár J, et al. Molecular testing in lung cancer in the era of precision medicine. Transl Lung Cancer Res. 2014;3(5):291-300.

11. De Leyn P, Dooms C, Kuzdzal J, et al. Preoperative mediastinal lymph node staging for non-small cell lung cancer: 2014 update of the 2007 ESTS guidelines. Transl Lung Cancer Res. 2014;3(4):225-233.

12. Herbst RS, Heymach JV, Lippman SM. Lung cancer. N Engl J Med. 2008;359(13):1367-1380.

13. de Groot PM, Carter BW, Betancourt Cuellar SL, et al. Staging of Lung Cancer. Clin Chest Med. 2015;36(2):179-196.

14. Shepherd FA, Crowley J, Van Houtte P, et al. The International Association for the Study of Lung Cancer lung cancer staging project: proposals regarding the clinical staging of small cell lung cancer in the forthcoming (seventh) edition of the tumor, node, metastasis classification for lung cancer. J Thorac Oncol 2007;2:1067-1077.

15. Roriz W. Tratamento cirúrgico do câncer de pulmão não pequenas células. In: Roberto Saad Junior [et al]. Cirurgia Torácica Geral. São Paulo: Editora Atheneu 2011.p. 411-418.

16. Nunes RA, Higa C, Saito EH, et al. Pré-operatório em cirurgia torácica geral. In: Silveira IC. O pulmão na prática médica. Rio de Janeiro Editora Epub 2000. p. 889-904.

17. Ribeiro-Netto A. A ressecção extramusculoperiostal "em gaiola de passarinho" (procedimento de Ribeiro-Netto) dos tumores pulmonares malignos invasores da fáscia costal da parede torácica, dos tumores primários ou secundários da parede torácica, do pulmão patológico e dos empiemas pleurais crônicos. [Dissertação]. Rio de Janeiro: Universidade do Estado do Rio de Janeiro; 1988.

18. Santos HT, Lopes AJ, Higa C, et al. Lung cancer with chest wall invasion: retrospective analysis comparing en-bloc resection and 'resection in bird cage'. J Cardio Thorac Surg. 2014 Mar 22;9:57.

19. Losso LC, Haddad R. Cirurgia torácica videoassistida. In: Roberto Saad Junior[et al]. Cirurgia Torácica Geral. São Paulo: 


\section{Artigo de revisão}

Editora Atheneu 2011.p. 639-652.

20. Roviaro GC, Rebuffat C, Varoli F, et al. Videoendoscopic Pulmonary Lobectomy for Cancer. Surg Laparosc Endosc. 1992;2:244-247.

21. McKenna RJ Jr, Houck W, Fuller CB. Video-assisted thoracic surgery lobectomy: experience with 1,100 cases. Ann Thorac Surg. 2006;81(2):421-425.

22. Lewis RL, Caccavale RJ, Sisler GE. One hundred video-assisted thoracic surgical simultaneously stapled lobestomies without rib spreading. Ann ThoracSurg. 1997;63:1415-22.

23. Naruke T. Thoracoscopic surgery for small, non-smal cell lung cancer. Is video assisted lobectomy an adequate treatment?
Pneumol. 1997;23:S17.

24. Daniels LJ, Balderson SS, Onaitis MW, et al. Thoracoscopic lobectomy: a safe and effective strategy for patients with stage I lung cancer. Ann ThoracSurg. 2002;74:860-4

25. Gharagozloo F, Margolis M, Tempesta B, et al. Robot-assisted lobectomy for early-stage lung cancer: report of 100 consecutive cases.Ann Surg. 2009;88(2):380-384.

26. Onishi H, Arakaki T, Shirato H, et al. Stereotactichypofractionated high-dose irradation for stage I nonsmallcelllung carcinoma: clinicaloutcomes in 245 subjects in a Japanesemultiinstitutionalstudy. Cancer 2004;1001:1623-1631. 